\title{
Efficient Boost Converter for Thermoelectric Energy Harvesting
}

\author{
J.-M. Gruber, S. Mathis \\ Institute of Embedded Systems (InES), \\ Zurich University of Applied Sciences (ZHAW) \\ Winterthur, Switzerland \\ gruj@zhaw.ch
}

\begin{abstract}
This paper presents a new boost converter for thermoelectric energy harvesting. The output voltage is regulated at $3.6 \mathrm{~V}$ for use with embedded systems. The booster reaches high efficiency at low input voltages of few $10 \mathrm{mV}$. A first prototype operates at about $15 \mathrm{mV}$ input voltage with an efficiency of $45 \%$. At $120 \mathrm{mV}$ input voltage the efficiency increases to $74 \%$. Using a $30 \times 30 \mathrm{~mm}$ sized low cost thermoelectric generator (TEG) in combination with the new developed booster, enough energy can be harvested to power an embedded wireless sensor.
\end{abstract}

Key words: DC-DC converter, thermal energy harvesting, low input voltage, thermoelectric generator, TEG, boost converter

\section{Thermal Energy Harvesting}

In environments with heat waste, thermoelectric generators (TEG) can be used to convert thermal energy in electrical energy. Typical applications are heat radiators or pipes with hot fluids. Also heat from mechanical or electrical equipment is a potential energy source, e.g. industrial drives. The conversion process is based on the Seebeck effect. The output voltage is proportional to the temperature difference.

\section{Motivation}

Small TEGs provide an output voltage of a few $10 \mathrm{mV}$ at small temperature differences. A boost converter is needed to convert the voltage into a usable voltage level for sensors and embedded systems. It should be able to start up and operate efficiently at these low voltages. Under these conditions, commercial available boost converters operate with low efficiency or don't operate $[1,2,3]$.

Goal of this work is to realize a boost converter which can directly start and operate at high efficiency from several $10 \mathrm{mV}$. In order to supply sensors and electronics, the output voltage is adjustable up to $3.6 \mathrm{~V}$. Costs are comparable to commercial available solutions.

\section{Booster Layout}

Fig. 1 shows the simplified block diagram of the booster. The TEG input is on the left and the regulated output voltage on the right side.

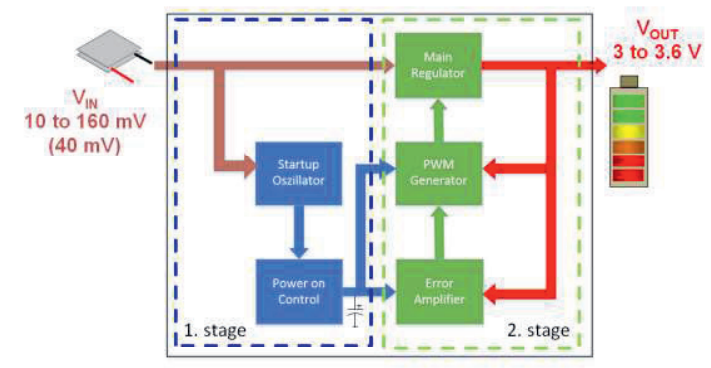

Fig. 1 Simplified block diagram of the developed boost converter

The converter has two separated stages. The first stage is used to start up the converter from low input voltages. The first stage starts charging an intermediate capacitor. When the capacitor is charged, the collected energy is used to activate the second stage. The second stage operates with high efficiency and provides a regulated output voltage of $3.6 \mathrm{~V}$.

\section{Startup}

The first stage is a startup circuit for cold start. It operates at an input voltage of about $36 \mathrm{mV}$. The startup circuit is a self-resonant oscillator connected to a voltage doubler. It is shown 
simplified in Fig. 2. The TEG is connected at the $V_{\text {IN }}$ node and the energy collected from the TEG is stored in the capacitor $C_{1}$ at the output.

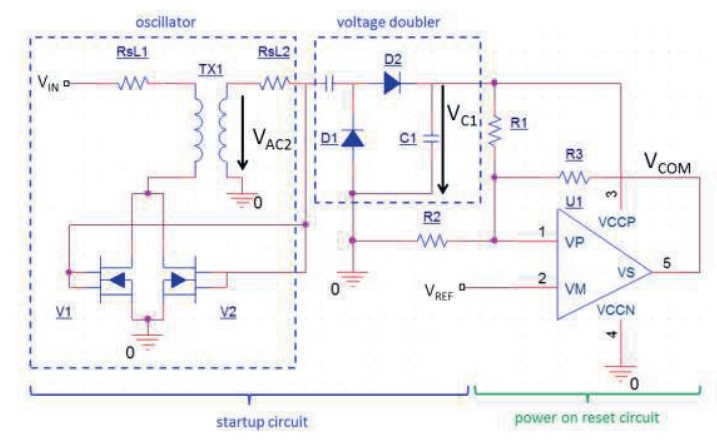

Fig. 2 First stage consisting of self-resonant oscillator, voltage doubler and power on reset

A coupled inductor in conjunction with two transistors builds an oscillator. The use of depletion FETs allows the converter to start from input voltages below the threshold voltage of enhancement FETs because conduction occurs at zero voltage. The parasitic gate capacitors of $V_{1}$ and $V_{2}$ and the secondary coil winding of TX1 form a series resonant circuit with a resonance frequency $f_{R}$ according to (1)

$$
f_{R}=\frac{1}{2 \pi \sqrt{L_{2} * C_{g t o t}}}
$$

Where $L_{2}$ is the secondary inductance of TX1 and $\mathrm{C}_{\text {gtot }}$ represents the sum of the gate capacitors of $V_{1}$ and $V_{2}$. Due to oscillation, an alternating voltage $V_{A C 2}$ is formed on the secondary winding. On the one hand $V_{A C 2}$ is fed back to $V_{1}$ and $V_{2}$ to sustain the oscillation and on the other $V_{A C 2}$ is rectified by the voltage doubler to charge the intermediate capacitor $C_{1}$.

The coupled inductor is realized by a transformer with a secondary inductance of $75 \mathrm{mH}$. RsL1 and RsL2 are the winding resistance of the primary and secondary coil in the simulation. While $\mathrm{C}_{1}$ is charged through the startup circuit, the power on reset circuit on the right side in Fig. 2 senses the voltage $V_{C_{1} \text {. The }}$ power on reset circuit consists of a shunt voltage reference $V_{R E F}$ and a comparator $U_{1}$ with three external resistors. With the voltage reference set to $1.25 \mathrm{~V}$ the trip points for $\mathrm{V}_{\mathrm{C} 1}$ become $\mathrm{V}_{\mathrm{TH}_{+}}=2.8 \mathrm{~V}$ and $\mathrm{V}_{\mathrm{TH}_{-}}=1.9 \mathrm{~V}$. The comparator output is low while $\mathrm{C}_{1}$ is charged but switches into high state as soon as $V_{\mathrm{C} 1}$ reaches $2.8 \mathrm{~V}$.

The startup circuit is optimized for low input voltages. Thus the overall efficiency of the startup circuit is about $9.5 \%$.
The startup circuit needs 73.1 seconds to charge a $10 \mu \mathrm{F}$ capacitor to $2.8 \mathrm{~V}$ with a measured output power of average $5.6 \mu \mathrm{W}$. Although the startup circuit is not suitable for high efficiency conversion, it collects enough energy to start the second stage.

\section{High Efficiency}

When the voltage on the intermediate capacitor $\mathrm{C}_{1}$ reaches $2.8 \mathrm{~V}$, the power on reset circuit activates the second stage. It consists of the main converter, a triangle wave generator, comparator and an error amplifier (Fig. 3). By turning on the main regulator control blocks, a pulse width modulated (PWM) signal is generated on the N-FET $V_{1}$, which turns on the main regulator, current flows through the inductor $\mathrm{L}$ and thus charges the output capacitor $\mathrm{C}_{\text {out }}$ to $3.6 \mathrm{~V}$.

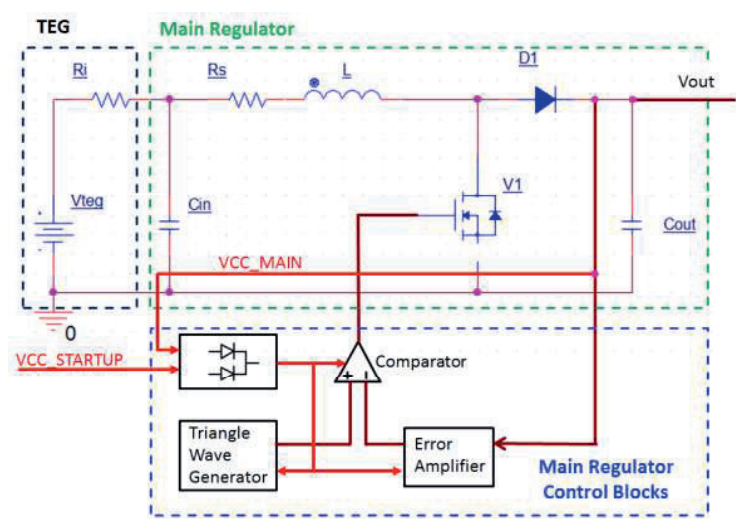

Fig. 3 Second stage with main regulator, control blocks and TEG on the left side

An error amplifier in voltage controlled mode is used for the output voltage regulation. The relaxation oscillator with a switching capacitor of $100 \mathrm{pF}$ generates the triangle wave signal. The triangle wave signal is compared with the error amplifier output to form the PWM signal. Due to high a voltage gain up to 200 , the converters duty cycle had to be equal $99 \%$ or more in continuous conduction mode. Instead, the converter works in discontinuous conduction mode with a maximum duty cycle limited to $66 \%$. Thereby the converter sets the closed loop input voltage approximately to $50 \%$ of the open loop TEG voltage if the output is loaded.

The new boost converter is tuned to operate in optimum efficiency with an evaluated TEG at low temperature differences. Internal resistance of the TEG is about $0.85 \Omega$. This combination starts harvesting energy at $1.5 \mathrm{~K}$ temperature difference. At $3 \mathrm{~K}$, the open loop output voltage on the TEG becomes $78 \mathrm{mV}$. Due to low TEG internal resistance, an input current of more than $44 \mathrm{~mA}$ must flow through the converter to 
achieve maximum power transfer between TEG and converter. In simulation of the main converter, a switching frequency of $5 \mathrm{kHz}$ on $\mathrm{V}_{1}$ in combination with an inductor $L$ of $36 \mu \mathrm{H}$ performed best in terms of overall efficiency.

For the inductor, simulation results with series resistances of $20 \mathrm{~m} \Omega$ or less showed good results. At $40 \mathrm{mV}$ input voltage, peak switching currents on the inductor up to $130 \mathrm{~mA}$ at full load are required. The TEG is not able to deliver the peak current directly. Input buffering capacitors are used. To reduce converter complexity and costs, a schottky diode D1 is used for rectification. Fig. 4 shows the component losses by performing a transient simulation of the main regulator. The TEG open loop voltage $V_{\text {TEG }}$ is set to $78 \mathrm{mV}$ to simulate a temperature difference of $3 \mathrm{~K} . \mathrm{C}_{\mathbb{I N}}$ and $\mathrm{C}_{\mathrm{OUT}}$ are pre-charged according to in- and output voltage. The output voltage of the converter is fixed at $3.6 \mathrm{~V}$ using a DC source.

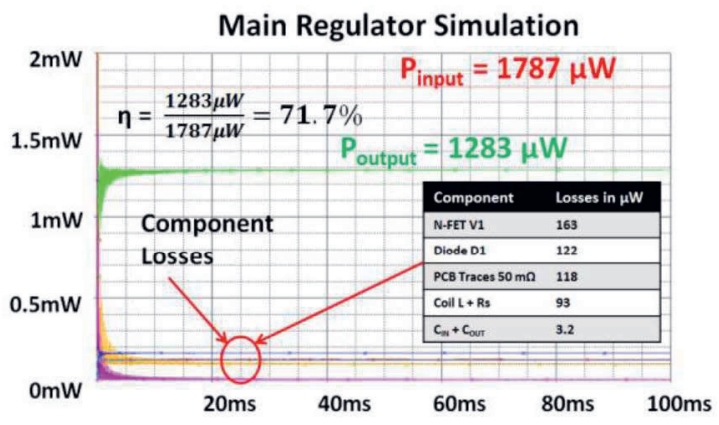

Fig. 4 Simulation results and component losses at $40 \mathrm{mV}$ input voltage ( $3 \mathrm{~K}$ on the TEG)

The simulated converter efficiency is about $71 \%$ at $40 \mathrm{mV}$ input voltage. Most of the losses are caused by $V_{1}$ and $D_{1}$, especially due to switch node ringing when turning off $\mathrm{V}_{1}$. For simulation, a PCB copper trace resistance of 50 $\mathrm{m} \Omega$ has been assumed. According to Fig. 4 this resistance already causes $118 \mu \mathrm{W}$ or $7 \%$ losses. Keeping PCB traces in the main regulator paths as short as possible reduces losses.

The real converter efficiency is lower compared to simulation. Supplying all control blocks including switching of $\mathrm{V}_{1}$ causes losses of 50.4 $\mu \mathrm{W}$. This drops the converter efficiency down to $68 \%$.

\section{Prototype}

The prototype PCB outlines measures $55 \times 60$ $\mathrm{mm}$ with a total height of $25 \mathrm{~mm}$ due to two $1500 \mu \mathrm{F}$ capacitors (Fig. 5).

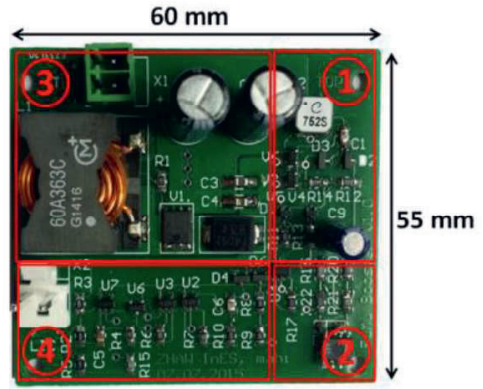

Fig. 5 Converter prototype assembled on a PCB

The converter is made of discrete components and functional blocks are arranged into four different blocks. In block 1 the startup oscillator with the voltage doubler and intermediate capacitor is shown. Block 2 contains the power on reset circuit which activates the main regulator as soon as the intermediate capacitor is charged to $2.8 \mathrm{~V}$. The components of the main regulator in block 3 are kept close to each other to reduce copper trace resistance. Main regulator control blocks such as error amplifier and PWM generator are located at the bottom in block 4.

\section{Prototype Results}

The converter was tested using a $30 \times 30 \mathrm{~mm}$ TEG with an internal resistance of $0.85 \Omega$ and Seebeck coefficient of $26 \mathrm{mV} / \mathrm{K}$. It was mounted between a labor hot plate and heat sink. In Fig. 6 the startup and operation of the developed converter is shown.

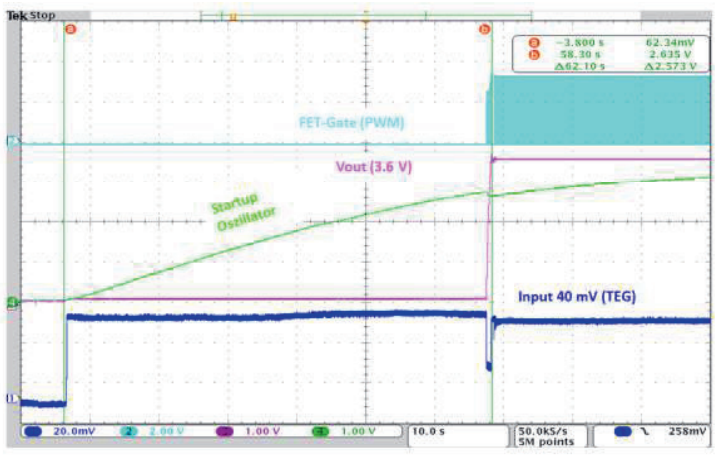

Fig. 6 Startup of the converter from TEG with $40 \mathrm{mV}$ input voltage

After reaching a stable output voltage of approximately $40 \mathrm{mV}(\mathrm{CH} 1$, dark blue) on the TEG, the converter was connected. Immediately the startup oscillator starts charging the intermediate capacitor $(\mathrm{CH} 4$, green). To power the control blocks during main regulator startup, the capacitor on the developed prototype was increased from $10 \mu \mathrm{F}$ to $47 \mu \mathrm{F}$. At $2.8 \mathrm{~V}$ the second stage is activated through the power on reset circuit which enables the PWM generator ( $\mathrm{CH} 4$, light blue) to start conversion on the main regulator. 
Charging the output capacitors to $3.6 \mathrm{~V}(\mathrm{CH} 3$, red) causes a short voltage drop on the input voltage. Approximately one minute elapsed until $3.6 \mathrm{~V}$ was measured at the output. Although no load was connected, the input voltage is slightly lower compared to the startup phase due to main regulator control block supply.

\section{Comparison of Different Combinations}

The developed booster in combination with TEG 071-150-22 (30x30 mm) and TEG 049$150-30(25 \times 25 \mathrm{~mm})$ is compared against other combinations with commercial available converters. Three boost converters in total, LTC3108, ECT310 and BQ25504 are tested with different types of TEGs at temperature differences from 3 to $15 \mathrm{~K}$ on the TEG. The results of the measurements are shown in Fig. 7. On the LTC3108 and ECT310 the TEG internal resistance is important for maximum power transfer. While the ECT310 requires a TEG with less than $2 \Omega$ [1], the LTC3108 equivalent input impedance depends on input voltage while VOUT is charging [2]. A TEG with an internal resistance of $6.6 \Omega$ and Seebeck coefficient of $56 \mathrm{mV} / \mathrm{K}$ was chosen and a transformer ratio of $1: 20$ on the LTC3108. On the BQ25504, the TEG impedance does not matter because of maximum power point tracking [3]. For all measurements, the output voltage of the converters was set to $3.3 \mathrm{~V}$.

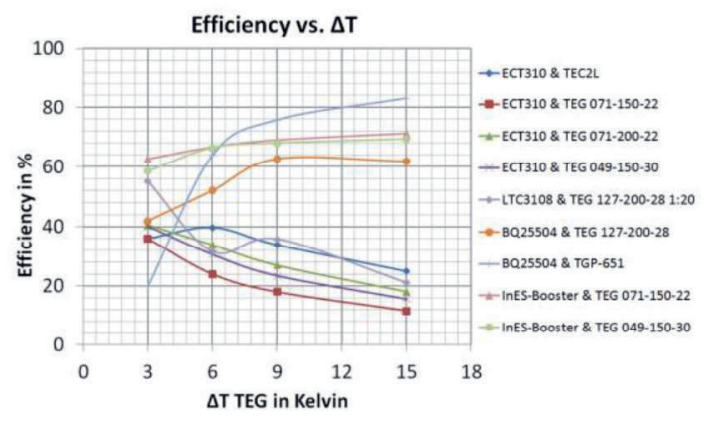

Fig. 7 Efficiency comparison of different combinations

At a temperature difference of $3 \mathrm{~K}$, a combination efficiency of $62 \%$ was achieved with the developed converter while delivering $802 \mu \mathrm{W}$ at the output. Similar results were measured on the LTC3108, about 55\% with constant output power of $719 \mu \mathrm{W}$. With increased temperature difference, the efficiency on the LTC3108 and ECT310 drops due to output voltage limitation on the transformer. In contrast, the efficiency on the developed converter and BQ25504 increases with higher input voltage because conversion takes place according to boost instead of flyback topology.

\section{Conclusion}

A solution is presented for thermoelectric energy harvesting at very low input voltages. The new developed boost converter reaches high efficiency at $15 \mathrm{mV}$ input voltage. The converter can start directly from a small TEG with $36 \mathrm{mV}$ input while delivering a regulated output voltage of $3.6 \mathrm{~V}$. A prototype has been realized and tested. At $3 \mathrm{~K}$ temperature difference on a small TEG the prototype reached an efficiency of $62 \%$ while delivering $802 \mu \mathrm{W}$ of power.

\section{References}

[1] EnOcean GmbH, „www.enocean.com,“ 32012. [Online]. Available:

https://www.enocean.com/de/enocean_module/e ct-310-perpetuum-data-sheet.pdf. [Access 3003 2017].

[2] Linear Technology Corporation, „www.linear.com," 2010. [Online]. Available: http://cds.linear.com/docs/en/datasheet/3108fc.p df. [Access 3003 201].

[3] Texas Instruments Incorporated, „www.ti.com, “ 6 2015. [Online]. Available: http://www.ti.com/lit/ds/symlink/bq25504.pdf. [Access 3003 2017]. 